\title{
Estudio comparativo de la mineralogía y los coloides arcillosos en suelos de trópicos húmedos
}

\author{
Comparative study of mineralogy and clay colloids in the \\ humid tropical soils
}

Juan Bullón Ames', Erika Bullón Castillo²

Universidad Continental

\section{RESUMEN}

Objetivos: Comparar las diferencias de la composición mineralógica de los suelos de Malasia y Zaire con suelos del Perú, en relación al tipo de coloides arcillosos que poseen. Métodos: Se utilizó el método analítico descriptivo correlacional, siendo las variables: a) Tipo de arcillas, b) Propiedades físicas del suelo y c) Propiedades químicas. Se operacionalizó las variables siendo sus indicadores: Para tipo de arcillas: kaolinitas, halloysitas, Illitas, cloritas y esmectitas; para Propiedades físicas, textura: arena, limo y arcilla; para propiedades químicas: $\mathrm{pH}$, aluminio cambiable, acidez cambiable $\mathrm{KCl}$, acidez cambiable $\mathrm{BaCl} 2 \mathrm{TEA}$. Para determinar estos indicadores se utilizaron los métodos analíticos siguientes: Termodiferencial y Microscopia electrónica para el tipo de arcillas y los minerales goetita y gibsita. Las propiedades físico-quimicas fueron determinadas por los siguientes métodos: La Granulometría (textura) por el método Bouyoucos, $\mathrm{pH}$ por potenciometría, el aluminio cambiable por titulación del extracto con $\mathrm{KCl}$, acidez cambiable $\mathrm{KCl}$ por el método Yuan, y acidez cambiable $\mathrm{BaCl} 2$ TEA por Cloruro de bario. Resultados: Se encontró que los Oxisoles están compuestos por arcillas Kaolinitas y por los minerales: Gibsita y Goetita, mientras que los Ultisoles están conformados por arcillas Illiticas, complejos expandibles esmectíticos, en menor proporción por Kaolinitas. Conclusión: Los Ultisoles presentes en Perú son más fértiles que los Oxisoles.

Palabras claves: Coloides, oxisoles, ultisoles, mineralógica, termodiferencial, kaolinitas, gibsita, goetita, illitas.

1 Ph.D, M.Sc, Ing. Agrónomo, docente universitario, gerente B-C Consultora Constructora Ingenieros S. A. C.

2 Bachiller en Ciencias Agronómicas, consultora. B-C Consultora Constructora Ingenieros S. A. C. 


\section{ABSTRACT}

Objectives: To compare the differences between the Malaysia and Zaire and the Peru's soils mineralogical composition, in relation to the clayey colloids type they own. Methods: We used the correlational descriptive analytical method, with the variables: a) Clay type, b) Soil physical properties and c) Chemical properties. Variables were operationalized, being its indicators: For clay type: kaolinites, halloysites, illite, chlorite and smectite; for Physical properties, texture: sand, silt and clay; for chemical properties: $\mathrm{pH}$, exchangeable aluminum, exchangeable acidity $\mathrm{KCl}$, exchangeable acidity $\mathrm{BaCl} 2$ TEA. To determine these indicators, the following analytical methods were used: thermodifferential and electron microscopy for the clay type and goethite and gibbsite minerals. The physico-chemical properties were determined by the following methods: The granulometry (texture) by Bouyoucos method, $\mathrm{pH}$ by potentiometry, the exchangeable aluminum by extract titration with $\mathrm{KCl}, \mathrm{KCl}$ exchangeable acidity by Yuan method, and exchangeable acidity $\mathrm{BaCl} 2 \mathrm{TEA}$ by Barium chloride. Results: Oxisols are composed by Kaolinites clays and by the gibbsite and goethite minerals, while Ultisols are formed by Illiticas clays, smectitic expandable convoluted, in a lesser proportion by kaolinites. Conclusions: Ultisols located in Peru are more fertile than Oxisols.

Keywords: Colloids, oxisols, ultisols, mineralogical, thermodifferential, kaolinites, gibbsite, goethite, illite.

\section{INTRODUCCIÓN}

El problema central de esta investigación es el desconocimiento de los componentes mineralógicos y coloidales de suelos de selva alta y baja (Perú) compuestos por Oxisoles y Ultisoles comparados con suelos de trópicos húmedos de Malasia (Asia) y de Zaire (África).

En los antecedentes se encontró que el estudio de la mineralogía de los suelos, Oxisoles y Ultisoles de los trópicos húmedos, ha sido objeto de numerosas investigaciones a nivel mundial $(1,2)$, una de las conclusiones que se ha desprendido de la revisión bibliográfica es que, existen varios resultados de la composición mineralógica de Oxisoles y Ultisoles $(3,4)$, en el presente trabajo se ha determinado las propiedades físico-químicas-coloidales presentes en los minerales de los suelos estudiados de Perú, Malasia y Zaire para conocer los diferentes tipos de arcillas y los minerales que componen cada uno de estos suelos, de tal manera que sea posible solucionar el problema central de este trabajo, en lo relativo a fertilización balanceada de café y cacao en suelos de Selva Alta y Baja de Perú $(5,6,7)$ se ha realizado correlación y regresión lineal y exponencial entre las propiedades físico-químicas indicadoras de la riqueza de los suelos.

Se discuten los resultados de un trabajo de investigación realizado en tres suelos peruanos, dos de Malasia y uno africano.

Con la finalidad de comparar el tipo de arcillas presentes en suelos de los órdenes taxonómicos Ultisoles y Oxisoles y la correlación con las propiedades físico-químicas mencionadas, partiendo del conocimiento que las kaolinitas son las arcillas más pobres, por tener baja capacidad de adsorción de cationes, debido a que sus cargas electrostáticas negativas varían de 3 a $10 \mathrm{me} / 100 \mathrm{~g}$ de arcilla $(8,9)$, siendo importante porque la Amazonía peruana posee, Ultisoles y en menor proporción Oxisoles.

Este estudio permitió conocer la riqueza potencial de los Ultisoles frente a los Oxisoles. Para planificar el aprovechamiento productivo de los suelos de los trópicos húmedos del Perú. La caracterización de suelos permitirá identificar la capacidad de retención de cationes $(\mathrm{Ca}++, \mathrm{Mg}++, \mathrm{K}+$, $\mathrm{NH} 4+$ y microeementos catiónicos) que están presentes en los fertilizantes y materia orgánica aplicada $(8,9)$. Se ha encontrado que existen propiedades físico-químicas y coloidales en los dos órdenes de suelos 
estudiados, tales como: granulometría, $\mathrm{pH}$, Aluminio cambiable, Acidez cambiable y tipo de arcillas que son importantes para programar una fertilización químicoorgánica balanceada en Ultisoles y Oxisoles $(10,11)$.

\section{MATERIAL Y MÉTODOS}

El estudio comprendió tres partes: La primera, revisión de literatura especializada. La segunda describe los métodos analíticos utilizados y sitúa a los suelos en un contexto geográfico sistemático. La tercera parte discute los resultados que presentan las propiedades físico-químicas-coloidales y la mineralogía de todos los suelos elegidos.

Respecto a los materiales, se ha seleccionado dos Oxisoles de Malasia (series Segamat y Kuantan) y una muestra de Zaire África (serie S2QA-F10, he), tres muestras de suelos del orden Ultisoles de la selva tropical peruana (12), cuyas principales características son:

Oxisoles. Serie Segamat, Typic Haplortox La serie Segamat ocupa pequeñas superficies repartidas en varios lugares del territorio de Malasia, el clima es cálido y húmedo (precipitación de $3038 \mathrm{~mm}$ y temperatura media de $25^{\circ} \mathrm{C}$ ), el perfil del suelo es profundo de color variable de rojo $(2,5$ YR $4 / 6)$ a rojo amarillo (5 YR 5/8), desarrollado sobre andesitas, la textura es arcillosa, con buen drenaje (13). Serie Kuantan, Typic Acrorthox La serie Kuantan ocupa una pequeña superficie al Este de Malasia, (10.000 has), el clima es caracterizado por una precipitación anual de $3038 \mathrm{~mm}$ y una temperatura media de $24^{\circ} \mathrm{C}$. Serie S2QA-F10 he, Arenic Umbriustox. La serie Zaire está desarrollada sobre materiales de arena cuarcítica, estos materiales originales son de textura areno arcillosa, la precipitación anual es de $1700 \mathrm{~mm}$, la duración de la estación seca es de 2 a 3 meses, la temperatura anual varía entre 20 y $32^{\circ} \mathrm{C}(14)$.

Ultisoles. Se ha seleccionado 3 Ultisoles, de Perú; del departamento de San Martín (series: Bella Vista Alta, Tarapoto y La Habana) Serie
Bella Vista Alta, Typic Haplustult Pertenece a una zona forestal de relieve plano, sobre una terraza alta, la precipitación es de $1623 \mathrm{~mm}$ y una temperatura media anual de $26{ }^{\circ} \mathrm{C}$. El suelo reposa sobre un conglomerado dominado por granodiorita, los colores varían de gris rojizo oscuro (5 YR $4 / 2$ ) a marrón rojizo (5 YR 4/3). El drenaje es imperfecto (14). Serie Tarapoto, Oxic Haplustult Ocupa una importante extensión alrededor de la ciudad de Tarapoto, en una zona de relieve ondulado a plano. Desarrollado sobre terrazas medias a altas, el material original es arenisca ácida, los colores del suelo varían de marrón oscuro (7,5 YR 3/2) a marrón vivo (7,5 YR 5/8), son suelos bien drenados (15). Serie La Habana, Aeric Plinthic Paleustult. Es un suelo desarrollado sobre una terraza media a partir de un material arcilloso muy alterado proveniente de lutitas ácidas, los colores varían de marrón (10 YR 5/3) a gris rojizo (7,5 YR 7/2), el drenaje es malo y el suelo está cubierto por una vegetación forestal pantanosa.

Respecto a los métodos utilizados, se ha utilizado el método analítico, descriptivo, correlacional, siendo las variables investigadas: Tipo de arcillas, propiedades físicas del suelo y propiedades químicas del suelo. Estas variables han sido determinadas a través de los métodos (15) siguientes:

- Granulometría. La textura ha sido analizada por el método Bouyoucos, utilizando como dispersante exametafosfato de sodio.

- $\mathrm{pH}$. Ha sido determinado por potenciometría en una suspensión suelo-agua $1: 1$, y en una suspensión $1: 1$ suelo- $\mathrm{KCl}(1 \mathrm{~N})$, con un electrodo de vidrio (USDA-SCS, 1967).

- Acidez y aluminio Intercambiables. De acuerdo al método de Yuan (1959) las muestras son saturadas con una solución de $\mathrm{KCl}(1 \mathrm{~N})$. La acidez cambiable es titulada con $\mathrm{NaOH}$ en presencia del indicador fenolftaleína (15).

- Óxidos de Fierro Libre. Se determinan de 
acuerdo al método de Mehra y Jackson (1960), la extracción de fierro libre es mediante la técnica del ditionito-citratobicarbonato de sodio, la cual es realizada con el ácido sulfo salicílico (16).

- Capacidad de intercambio catiónico (CIC). Se determinó mediante el método del $\mathrm{NH} 4 \mathrm{Cl}(1 \mathrm{~N})$ no tamponado: la solución del suelo se saturó con $\mathrm{NH} 4$, posteriormente se desplazó el amonio con una solución de $\mathrm{KCl}$ al 10\% y se tituló con $\mathrm{HCl}$.

- Determinación mineralógica, de los coloides del suelo Mineralogía de la fracción fina $<2 \mu$ Análisis de las propiedades mineralógicas y coloidales de las muestras de los suelos Oxisoles y Ultisoles.

- Análisis termodiferencial. El análisis termo diferencial ha sido realizado en las muestras de arcilla de los horizontes óxicos y argílicos. Para lo cual se ha utilizado un aparato termo-analizador automático DUPONT 900. La interpretación de este análisis ha sido realizada de acuerdo a Mackenzie $(17,18)$.

- Microscopia electrónica. Las observaciones de la cristalografía de las arcillas y sus impurezas fueron realizadas mediante el microscopio electrónico, ello se practicó en las seis muestras de los suelos estudiados (3).

\section{RESULTADOS}

La tabla 1, muestra el resultado de los análisis físicos practicados por el método Bouyoucos o del hidrómetro, corresponde

Tabla N ${ }^{\circ}$ 1: Granulometría de los Oxisoles, método de Bouyoucos.

\begin{tabular}{|c|c|c|c|c|c|c|}
\hline Perfil & Hor. & $\begin{array}{c}\text { Profund. } \\
\mathrm{cm} .\end{array}$ & $\begin{array}{c}\text { Arena } \\
\%\end{array}$ & $\begin{array}{c}\text { Limo } \\
\%\end{array}$ & $\begin{array}{c}\text { Arcilla } \\
\%\end{array}$ & $\begin{array}{c}\text { Clase } \\
\text { textural }\end{array}$ \\
\hline & Al & $0-6$ & 1,8 & 7,2 & 91,0 & Arcillosa \\
\hline Segamat Typic & B 1 & $6-18$ & 1,8 & 7,2 & 91,0 & Arcillosa \\
\hline \multirow[t]{2}{*}{ Haplorthox } & B21 & 18-38 & 1,8 & 7,2 & 91,0 & Arcillosa \\
\hline & $\mathrm{B} 22 \mathrm{Ox}$ & $38-100$ & 1,7 & 7,9 & 90,4 & Arcillosa \\
\hline \multirow{3}{*}{$\begin{array}{l}\text { Kuantan } \\
\text { Acrorthox }\end{array}$} & $\mathrm{A} 1$ & $0-8$ & 4,0 & 14,8 & 81,2 & Arcillosa \\
\hline & $\mathrm{B} 1$ & $8-20$ & 4,0 & 14,8 & 81,2 & Arcillosa \\
\hline & $\mathrm{B} 210 \mathrm{x}$ & $20-60$ & 4,0 & 15,9 & 80,1 & Arcillosa \\
\hline S2QA-F10 he & Al & $0-28$ & 84,8 & 11,5 & 3,8 & Arena limosa \\
\hline Arenic & $\mathrm{A} / \mathrm{B}$ & $28-60$ & 83,9 & 9,6 & 6,5 & Arena limosa \\
\hline Umbriustox & $\mathrm{IIB} 2 \mathrm{Ox}, 1$ & $60-95$ & 68,8 & 4,6 & 26,7 & Limo arcillo arenoso \\
\hline
\end{tabular}

Tabla N 2: Granulometría de los Ultisoles, método Bouyoucos.

\begin{tabular}{lrrcccc}
\hline Perfil & Hor. & $\begin{array}{c}\text { Profund. } \\
\mathrm{cm} .\end{array}$ & $\begin{array}{c}\text { Arena } \\
\%\end{array}$ & $\begin{array}{c}\text { Limo } \\
\%\end{array}$ & $\begin{array}{c}\text { Arcilla } \\
\%\end{array}$ & $\begin{array}{c}\text { Clase } \\
\text { textural }\end{array}$ \\
\hline Bellavista Typic & $\mathrm{A} 1$ & $0-15$ & 56,0 & 14,8 & 14 & Limo arenoso \\
Haplustult & $\mathrm{A} 2$ & $15-21$ & 30,0 & 14,8 & 18 & Limoso \\
& A3m & $21-39$ & 56,0 & 15,9 & 22 & Limo arenoso \\
Tarapoto Oxic & $\mathrm{Ap}$ & $0-25$ & 4,0 & 14,8 & 28 & Limo arcillo arenoso \\
Haplustult & $\mathrm{B} 21 \mathrm{t}$ & $25-45$ & 4,0 & 14,8 & 38 & Arcillo arenoso \\
La Habana & $\mathrm{B} 22 \mathrm{t}$ & $45-60$ & 4,0 & 15,9 & 38 & Arcillo arenoso \\
Aeric Plinthic & $\mathrm{A} 1$ & $0-40$ & 84,8 & 11,5 & 12 & Limo arenoso \\
paleaquult & $\mathrm{B} \dagger$ & $40-70$ & 83,9 & 9,6 & 54 & Arcillosa \\
\hline
\end{tabular}




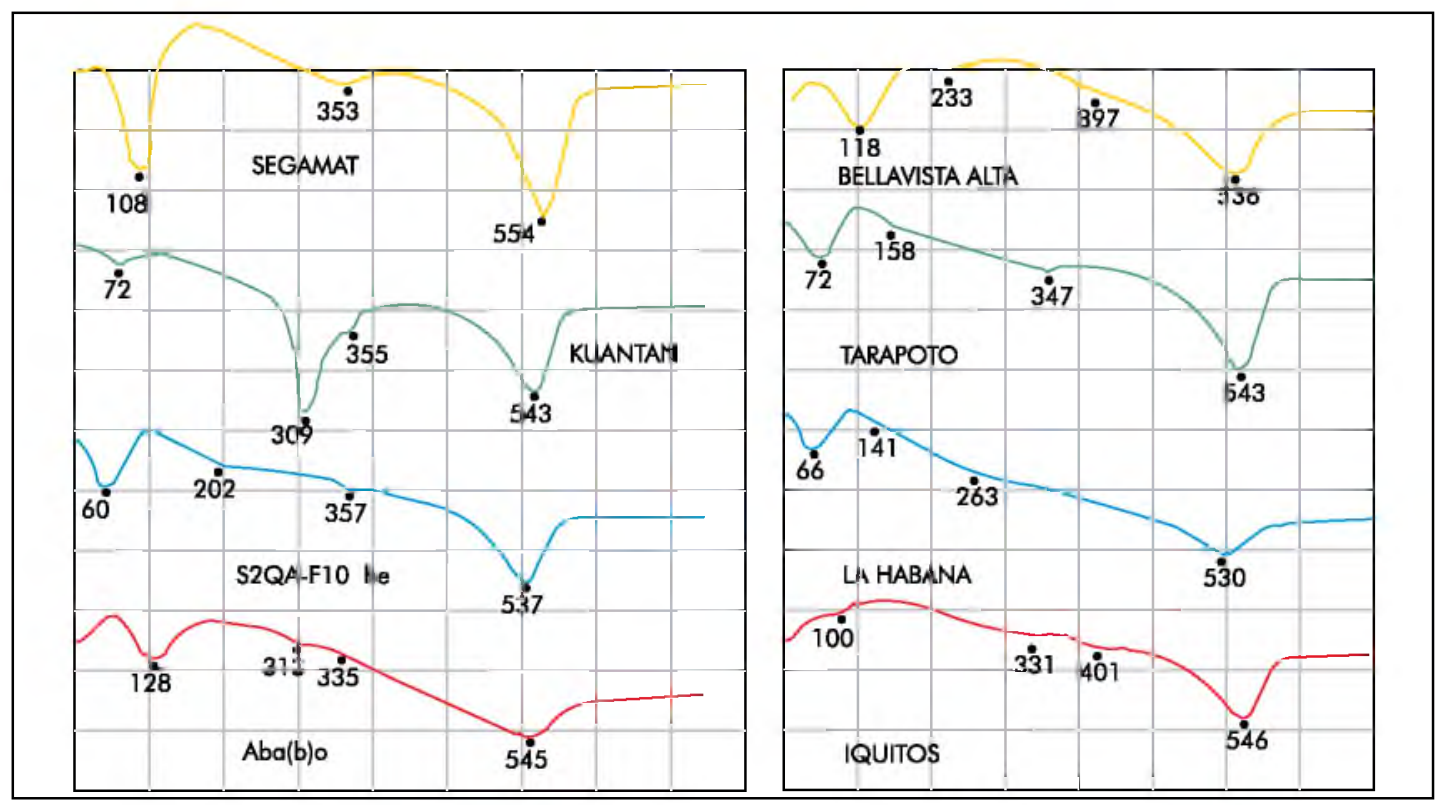

Figura $N^{\circ}$ 1: Análisis termo-diferencial en los horizontes óxicos y argílicos

a los horizontes de los perfiles de suelos Oxisoles de Malasia y de Zaire. Mientras que la tabla 2, presenta los resultados del análisis realizado en los horizontes de los tres perfiles de Perú, siendo suelos Ultisoles.

En la figura 1, se presenta los resultados del análisis termodiferencial de los Oxisoles con sus principales curvas de inflexión que corresponden a la deshidratación de las muestras de suelos expuestos a temperaturas variables desde $60{ }^{\circ} \mathrm{C}$ hasta $545^{\circ} \mathrm{C}$. Igualmente para los Ultisoles las temperaturas varían de 66 a $546^{\circ} \mathrm{C}$.
Las figuras 2 y 3 corresponden a la regresión y correlación entre la acidez cambiable $\mathrm{KCl}$ y el aluminio cambiable $\mathrm{KCl}$. Realizadas para los Oxisoles de Malasia y Zaire y para los Ultisoles de Perú.

Las figuras 4 y 5 , representan las regresiones y correlaciones estadísticas de comprobación de los resultados entre los indicadores acidez cambiable $\mathrm{BaCl} 2$ TEA y aluminio cambiable $\mathrm{KCl}$.

Las figuras 6 y 7 muestran las regresiones y correlaciones estadísticas para Oxisoles y

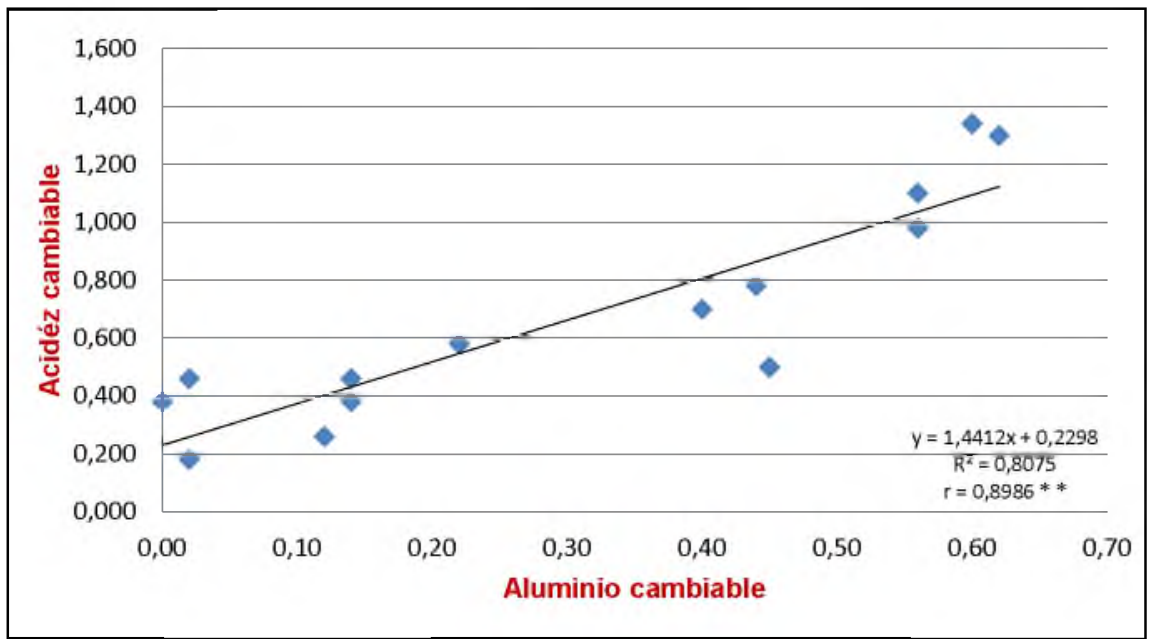

Figura $\mathrm{N}^{\circ}$ 2: Regresión y correlación lineal simple entre acidez cambiable y aluminio cambiable en oxisoles. 


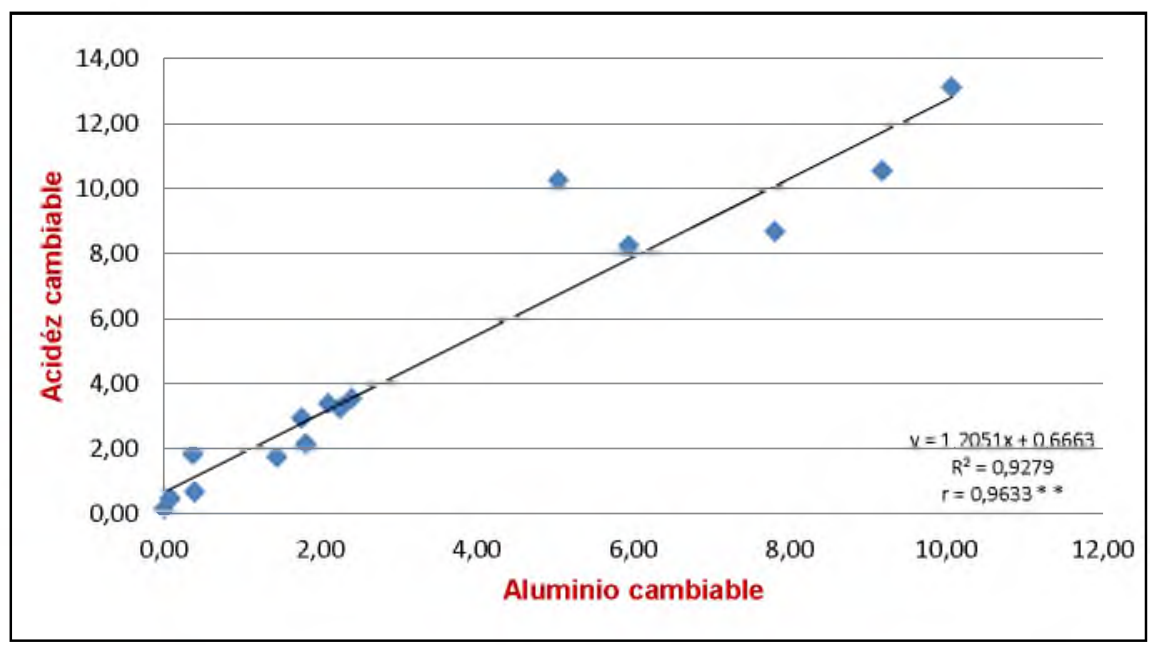

Figura $\mathrm{N}^{\circ}$ 3: Regresión y correlación lineal simple entre acidez cambiable y aluminio cambiable en ultisoles.

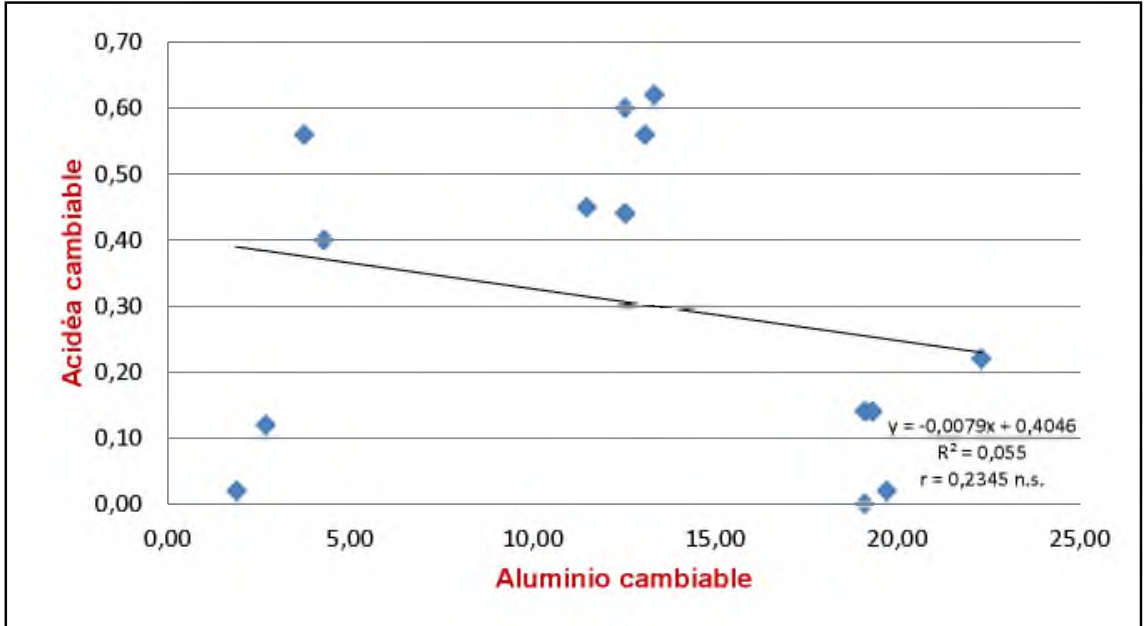

Figura $\mathrm{N}^{\circ}$ 4: Regresión y correlación lineal simple entre acidez cambiable $\mathrm{BaCl} 2, \mathrm{TEA}$ y aluminio cambiable en oxisoles.

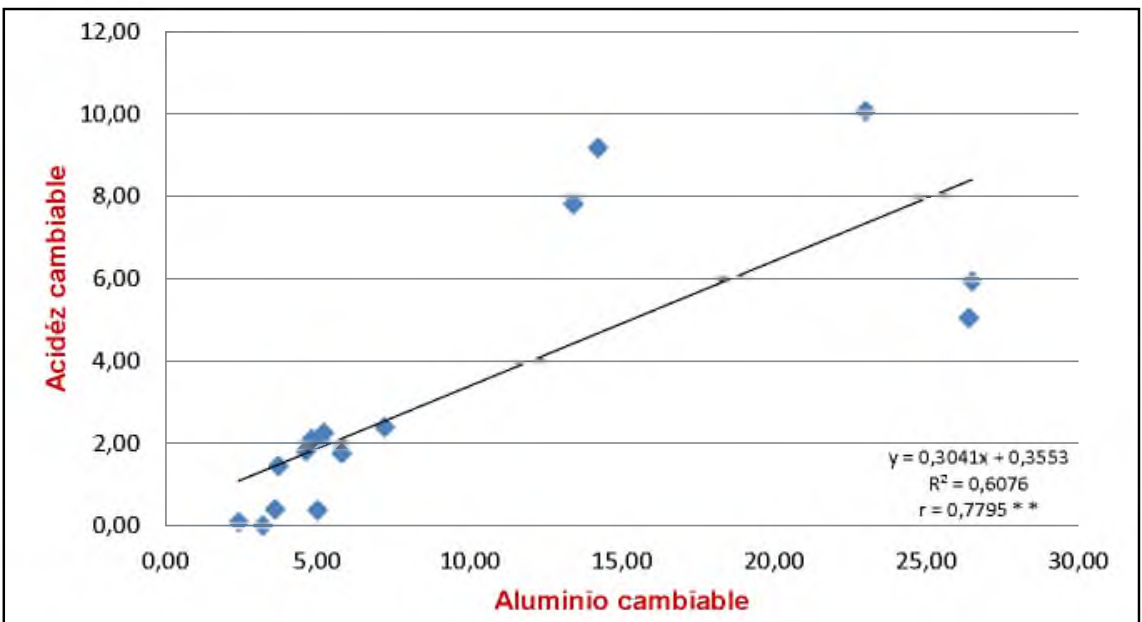

Figura $\mathrm{N}^{\circ}$ 5: Regresión y correlación lineal simple entre acidez cambiable $\mathrm{BaCl}$, TEA y aluminio cambiable en ultisoles. 


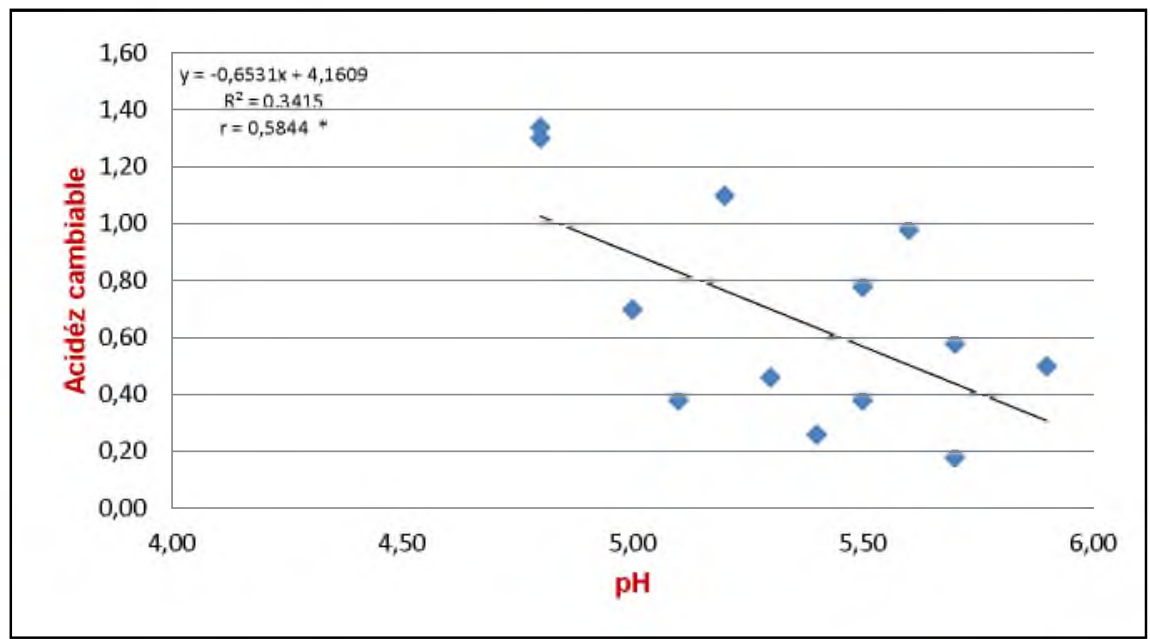

Figura $\mathrm{N}^{\circ}$ 6: Regresión y correlación lineal simple entre Acidez Cambiable $\mathrm{KCl}$ y pH H2O en Oxisoles.

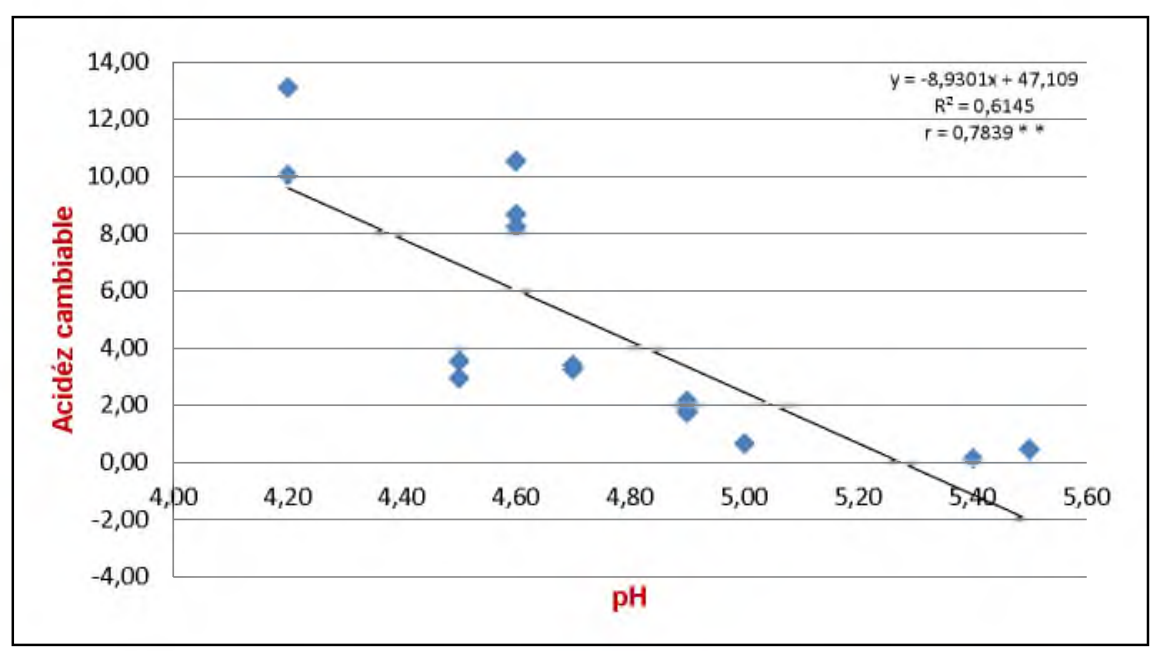

Figura $\mathrm{N}^{\circ}$ 7: Regresión y correlación lineal simple entre acidez cambiable $\mathrm{KCl}$ y $\mathrm{pH} \mathrm{H} 2 \mathrm{O}$ en ultisoles.

Ultisoles, comprobando la relación existente entre los indicadores citados de la variable propiedades físicas.

\section{DISCUSIÓN}

La granulometría se muestra en las tablas 1 y 2 . Todos los horizontes de los suelos Segamat y Kuantan son de textura arcillosa. La distribución de arcilla en los dos perfiles es homogénea y decrecen desde la superficie hacia abajo. La serie Segamat presenta un contenido de arcilla mayor que Kuantan. El porcentaje de limo aumenta en profundidad para los dos suelos. El contenido de arena es muy bajo en ambos suelos (15). En general todos los Ultisoles estudiados son de textura más gruesa que los Oxisoles derivados de rocas básicas. Variando en los horizontes superficiales de limo-arenoso a arena limosa. Todos los horizontes argílicos pertenecen a la clase arcillosa.

Composición mineralógica de la fracción $<2 \mu$. Análisis termodiferencial, las curvas termo diferenciales de los Ultisoles muestran tres inflexiones (reacciones endotérmicas) principales (figura 1). La primera es de intensidad baja a media y está situada entre 66 y $118^{\circ} \mathrm{C}$. Ella corresponde a la parte de agua (deshidratación) adsorbida a baja energía $(3,16)$. La segunda inflexión es muy baja y continúa a $380^{\circ} \mathrm{C}$, esta 
se debe a la deshidratación completa de los materiales illiticos, esmectíticos y los complejos illita-esmectita. La tercera inflexión es de intensidad fuerte y se sitúa entre 530 a $546{ }^{\circ} \mathrm{C}$, debida a la pérdida de agua de constitución (deshidroxilación) de la kaolinita. Hay una baja reacción endotérmica en la muestra del perfil Tarapoto a $347^{\circ} \mathrm{C}$ que es producida por la deshidroxilación de la Goetita (17). Las curvas termo diferenciales de los Oxisoles son más variadas que las curvas de los Ultisoles (figura $\mathrm{N}^{0} 1$ ). Estas presentan tres inflexiones. La primera de baja a fuerte intensidad, está situada entre 60 a 128 ${ }^{\circ} \mathrm{C}$, denota impurezas que acompañan a la kaolinita, así como la presencia de agua débilmente retenida por la kaolinita. La segunda reacción endotérmica es baja, se localiza entre 350 y $358{ }^{\circ} \mathrm{C}$ y es producida por la deshidroxilación de a Goetita. La tercera inflexión que es muy fuerte se sitúa a $540{ }^{\circ} \mathrm{C}$ y corresponde a la deshidroxilación de la kaolinita. El oxisol Kuantan muestra además una fuerte inflexión a $309{ }^{\circ} \mathrm{C}$ debido a la deshidroxilación de la Gibsita (16).

Microscopia electrónica. Las observaciones en los Oxisoles (Kuantan, Segamat y SE2QA-F10 he) (3) (18), mediante el microscopio electrónico muestran minerales gruesos bajo la forma hexagonal bien cristalizados (kaolinitas) y pequeños cristales de forma irregular (Goetita). En los Ultisoles Tarapoto y Bellavista Alta, estos minerales están presentes, pero los contornos de los cristales son des uniformes (19).

$\mathrm{pH}$. El $\mathrm{pH}$ en agua varía entre 4,8 a 5,9 en todos los horizontes de los Oxisoles. Siendo un poco más bajo en la superficie que en profundidad en las series Segamat y Kuantan. El $\mathrm{pH}$ en $\mathrm{KCl}$ es siempre inferior al $\mathrm{pH}$ en agua. (3) (20). La medida del $\mathrm{pH}$ en agua y en $\mathrm{KCl}$ permite reconocer la predominancia de hidróxidos libres sobre los óxidos en los aluminosilicatos, (20), figuras 6,7$)$. En los Ultisoles, el $\mathrm{pH}$ en agua varía de 3,9 a 5,5 y es ligeramente más elevado en la superficie que a profundidad contrariamente a los Oxisoles. De acuerdo a Mekaru y Uehara (1972) (21). Acidez cambiable $\mathrm{KCl}$. La acidez cambiable $\mathrm{KCl}$, en los tres Oxisoles es baja, ello está en relación a la baja cantidad de aluminio cambiable de esos suelos. La regresión y correlación lineal de la acidez cambiable en función del pH muestra una diferencia neta entre la acidez cambiable de los Ultisoles y de los Oxisoles (figuras 2, 3) (3), (21).

Aluminio cambiable. El tenor de aluminio cambiable es bajo en los tres Oxisoles. Las regresiones y correlaciones lineales simples de la acidez cambiable y el aluminio cambiable en $\mathrm{KCl}$, se presentan en las figuras 2 y 3 . En ambos suelos son positivas y altamente significativas.

En las figuras 4, 5 se muestran la regresión lineal simple de la Acidez Cambiable $\mathrm{BaCl} 2$, TEA y el Aluminio Cambiable KC entre los Oxisoles y los Ultisoles siendo negativa y no significativa en los Oxisoles, mientras que en los Ultisoles fue positiva y estadísticamente significativa. En las figuras 6,7 se muestra los resultados de la regresión y correlación lineal simple entre la acidez cambiable $\mathrm{KCl}$ y el $\mathrm{pH}$ en $\mathrm{H} 2 \mathrm{O}$, en Oxisoles y Ultisoles; donde la regresión y correlación es negativa en ambos suelos, significativa en los Oxisoles y negativa y altamente significativa en los Ultisoles. Ello indica que a medida que el $\mathrm{pH}$ aumenta la acidez cambiable disminuye en ambos suelos. Los Ultisoles presentan una concentración mayor de aluminio cambiable (22).

De los resultados se llegó a las conclusiones siguientes:

- Las curvas termo diferenciales de los Oxisoles son más variadas que las curvas de los Ultisoles (figura 1). Ello indica la diferencia de pérdida de agua por las altas temperaturas a la que se someten las arcillas investigadas. Los horizontes de los suelos Segamat y Kuantan son de textura arcillosa, los de Zaire son arenosos. Los Ultisoles estudiados presentan textura más gruesa que los Oxisoles.

- Las arcillas presentes en los Oxisoles son kaolinitas, en menor proporción goetitas y gibsitas, óxidos de fierro libre y de 
aluminio. En los Ultisoles las arcillas son Illitas, un poco de cuarzo, esmectíticas y kaoliníticas.

- El $\mathrm{pH}$ en $\mathrm{KCl}$ es inferior al $\mathrm{pH}$ en agua. En los suelos estudiados, por lo cual se puede calcular la carga negativa del coloide suelo.

- La regresión y correlación lineal de la acidez cambiable en función del $\mathrm{pH}$ muestra una diferencia neta entre la acidez cambiable de los Ultisoles y de los Oxisoles. En los Oxisoles la correlación lineal simple de la Acidez Cambiable $\mathrm{BaCl} 2$, TEA y el Aluminio Cambiable $\mathrm{KCl}$ fue negativa $y$ no significativa, mientras que en los Ultisoles fue positiva y estadísticamente significativa.

Estos hallazgos permiten concluir que los Ultisoles (Perú) presentan mayor potencial productivo que los Oxisoles (Asia y África).

\section{REFERENCIAS BIBLIOGRÁFICAS}

1. Arias F, Mata R. Mineralogía de la fracción arcilla de algunos suelos Cultivados con banano en las Ilanuras aluviales del Caribe de Costa Rica. Agronomía Costarricense. 2010; 34(2): 197-222.

2. Besoain E. Mineralogía de arcillas de suelos. San José: Bib. Orton IICA; 1985.

3. Bullón J. Etude comparative de la Mineralogie et de L'Adsorption de Phosphates et de Sulfates dans les sols Tropicaux. [Tesis doctoral]. Bélgica: Université d'Etat de Gand; 1976.

4. Brinkman R. Ferrolysis a hydromorphic soil forming process. Geoderma. 1970; 3: 199-206.

5. Cochrane $T$, Sánchez $P$ Recursos de tierras, suelos y su manejo en la región amazónica: Informe acerca del estado de conocimiento. Amazonía Investigación Agricultura Uso de Tierras. 1982; 4(82): 141-203.

6. Coleman N.T, Thomas G.W. The basic chemistry of soil acidity. Advances in Agronomy. 1967; 12: 1-41.

7. Correa J.C. Recursos edáficos do
Amazonas. Manaos: EMBRAPA - UEPAE; 1984.

8. Fassbender H.W. Química de suelos, con énfasis en suelos de América latina. 5a ed. San José: IICA; 1986.

9. Forsythe W. Física de suelos: Manual de laboratorio. San José: IICA; 1985.

10. Grim R.E. Clay Mineralogy. $2^{\mathrm{a}}$ ed. New York: Mc Graw-Hill; 1968.

11. Hashimoto I, Jackson M.L. Rapid dissolution allophone and kaolinitahalloysite after dehydration. Clays and Clay minerals. 1958; 7: 102113.

12. Hoag, R. Characterization of soil floodplains of tributaries flowing into the Amazon River in Perú. [Tesis doctoral]. Carolina del Norte: North Carolina State University; 1985.

13. Instituto Tecnológico Geominero de España. Contaminación y depuración de suelos. Madrid: ITGE; 1995.

14. Jackson M.L. Soil Chemical Analysis. Advanced course. Madison: UWMadison; 1956.

15. Krauskopf K.B, Bird D.K. Introduction to geochemistry. New York: McGraw-Hill: 1995.

16. Mackenzie R.C. The differential thermal investigation of clay minerals. London: Mineralogical Society; 1957.

17. Mclean J.E, Bledsoe B.E. Behaviour of metals in soils. Washington, DC: US EPA/Ground Water Issue; 1992. EPA/540/S-92/018.

18. Mehra O.P, Jackson M.L. Iron oxide removal from soils and clays by dithionite-citrate system buffered with sodium bicarbonate. Clay and Clay minerals. 1960; 7(88): 315-342.

19. Mejía M. Caracterización mineralógica de los suelos tropicales de la reserva forestal de la Universidad Agraria de la Selva por difractometría de rayos $\mathrm{x}$ y espectroscopia Mössbauer. [Tesis de pregrado]. Lima: Universidad Nacional Mayor de San Marcos; 2011.

20. Oficina Nacional de Evaluación de Recursos Naturales. Clasificación de las tierras del Perú. Lima: ONERN; 1982.

21. Rojas $A$. Criterios para la interpretación del análisis mineralógico de arcillas. Seminario-Taller "Fundamentos para 
la interpretación de análisis de suelos, plantas y aguas para riego". Bogotá: Sociedad Colombiana de la Ciencia del Suelo; 1991.

22. Yuan T.L. Determination of exchangeable hydrogen in soils by a titration method. Soil Sci. 1959; 88: 164-167. 